\title{
7 Dissoziation und dissoziative Erkrankungen im Kontext der transkulturellen Psychiatrie
}

Thomas Wenzel und Li ling Zhu

\subsection{Transkulturelle Psychiatrie und belastungsabhängige Erkrankungen als Rahmen für den Diskurs zu dissoziativen Phänomenen}

Die Auseinandersetzung mit anderen Kulturen und der Relativität eigener kultureller Modelle in der Psychiatrie hat eine lange und komplexe Vorgeschichte, für die Dissoziation als Phänomen und Krankheitsmodell als gutes Beispiel dienen kann (Degkwitz 1975).

Transkulturalität kann in diesem Zusammenhang in zweierlei Hinsicht verstanden werden:

1. als Auseinandersetzung mit den Modellen anderer Kulturen (die umgekehrt auch zu einer Reflexion der eigenen kulturabhängigen Modelle führen kann bzw. sollte)

2. oder als Beschäftigung mit den im Rahmen der gesellschaftlichen Entwicklungen wechselnden, kulturabhängigen Modellen von Krankheit, Gesundheit und Heilung.

Belastungsabhängige Erkrankungen sind in diesem Zusammenhang geeignet, um die Einbettung in die Kultur, die dynamische Entwicklung und die Interaktion mit dem sozialen Umfeld zu verdeutlichen. Im Rahmen der internationalisierten Modelle der ICD-1o der Weltgesundheitsorganisation (Degkwitz 1975) ist neben der posttraumatischen Belastungsstörung (PTBS) auch an dissoziative Störungen zu denken. 
Die Bedeutungsveränderung des heute infolge des historischen Paradigmenwechsel eher unklaren Begriffs der „Dissoziation“ (Isaac u. Chand 2006), der in der ICD 10 weitgehend im Kontext der älteren Begriffe der Hysterie und der Konversionsstörung gesehen wird, kann in diesem breiteren Kontext besser verstanden werden.

\section{2 „Sprachen“ der Belastung}

Im Rahmen der transkulturellen Psychiatrie findet hier zunehmend der Begriff der belastungsabhängigen kulturabhängigen Reaktionen oder der „Idiome der Belastung“ (,Idioms of distress“ [IOD] [van Duijl et al. 2010]) Anwendung. Diese beschreiben den vor allem von der Kultur geprägten Ausdruck von psychischer Belastung, Sorgen oder Konflikten auf verbaler oder nonverbaler Ebene. Sie entsprechen oft auch bestimmten Erwartungen der sozialen Umgebung an den Betroffenen und umgekehrt auch Erwartungen des Individuums an die Reaktion der Cruppe, die in einer Hilfestellung anderer im sozialen Netzwerk oder dem Verweis an bestimmte religiöse oder medizinische Experten besteht. So war es beispielsweise über viele Jahrhunderte in Europa üblich, in von sozialen Konflikten geprägten oder sonst belastenden Situationen kurz zusammenzusinken und unter Umständen sogar kurz das „Bewusstsein zu verlieren“ („faint“). In diesem Fall erfolgte meist sofortige Aufmerksamkeit, Zuwendung und Unterstützung, wie beispielsweise auch die Anwendung eines (Ammoniak) Riechfläschchens als Teil der Reaktion der Anwesenden (Veith 1965). Ein anderes gängiges Vorstellungsmodell war das des „vorzeitigen Alterns" bei tiefgehendem Erschrecken - das Haar ergraut oder wird über Nacht „schlohweiß“ (siehe z.B. in Schubert: Der greise Kopf, Winterreise, D 911, Text Wilhelm Müller). Nicht nur durch den wesentlichen Beitrag der psychoanalytischen Bewegung und ihre Entstehung im Wien der Jahrhundertwende kann die „Hysterie“ jedoch als ein wesentliches, kulturabhängiges und damit dynamischen Veränderungen unterworfenes Krankheitsmodell (Veith 1965; Mace 1992; Scull 2009) unserer eigenen Kultur in dieser Zeit gesehen werden. Diese war bis zum Ende des 19. Jahrhunderts hinein von alten, unter anderem auch hippokratischen und vorhippokratischen Modellen geprägt (Hare 1991). Es wurde dabei davon ausgegangen, dass entweder „vapours" (Ausdünstungen) oder die Bewegung des Organs selbst in Symptomen resultiert, was auch dazu führte, dass lange Zeit die Erkrankung als ausschließlich „weiblich“ gesehen wurde bzw. überwiegend nur bei Frauen diagnostiziert wurde. So fremd dieses Modell heute erscheint, ist zu berücksichtigen, dass alte Paradigmen und Konzepte - wie hier das des Uterus als krankem Organ bei heute als neurovegetativ und/oder Angstsymptomatik interpretierten Symptomen - erst langsam durch neue Modelle abgelöst werden. So kann man noch im frühen 20. Jahrhundert Schriften finden, die „folgerichtig“ als Intervention eine Hysterektomie empfehlen. Die schwerwiegenden Risiken und Belastungen für die Betroffenen bestanden allerdings in 
unzureichender Anästhesie und hohem Komplikationsrisiko als indirekte Folge des heute verworfenen Krankheitsmodells.

Dissoziation und die heute in der ICD-10 in ausdrücklicher Nachfolge des „Hysterie“begriffs als „dissoziativ“ eingestuften Phänomene des Verlusts der Integration zwischen Bewusstsein, Gedächtnis und Motorik waren dabei in unterschiedlichsten Definitionen seit zumindest den letzten Jahrzehnten des 19. Jahrhunderts ein wichtiges Thema des Diskurses in Medizin und Öffentlichkeit (Mace 1992; Jones u. Wessely 2004; Neill 1993). „Kriegszitterer“ und eine Reihe heute nicht mehr oder selten beobachteter belastungsabhängiger Symptome, die heute am ehesten entweder der PTBS, der Konversion bzw. den dissoziativen Störungen zuzuordnen wären, sind ebenfalls in diesem Kontext zu sehen. Syndrome ändern sich im Rahmen der Entwicklung einer Kultur und sind als Teil eines Dialogs zwischen der „öffentlichen“, „Alltagskultur“ und den medizinischen Systemen zu sehen.

Hypnose als neues medizinisches Therapiemodell aber auch als öffentliches Spektakel in Jahrmärkten und Séancen bediente sich dabei vor allem auch dissoziativer Mechanismen. Das besondere Interesse in den sich in dieser Zeit rasch entwickelnden psychologischen und psychotherapeutischen Schulen trug zu einer unterschiedlichen Interpretation und einer komplexen Entwicklung (Mace 1992) des Begriffes bei.

Die aktuelle Fassung des „Diagnostic and Statistical Manual of Mental Disorders“(DSM-5) der American Psychiatric Association bezieht erstmals systematisch kulturabhängige ,idioms of distress“ in das Diagnosesystem ein und ermöglicht erstmals eine auf qualitativen Forschungsmodellen beruhende kulturspezifische Diagnostik („Cultural Formulation Interview“). Dieses wird es unserem Eindruck nach in Zukunft ermöglichen, diesen wesentlichen Aspekt besser als derzeit im ICD-10-Modell zu berücksichtigen.

\subsection{Dissoziation und verwandte Phänomene in der Definition der aktuellen WHO Klassifikationen}

Das ICD 10 der Weltgesundheitsorganisation (Degkwitz 1975) fasst eine Reihe der historisch zuzuordnenden Begriffe in der Kategorie F44 zusammen, und weist auf den möglichen Zusammenhang mit traumatischen Lebenserfahrungen hin. Es definiert:

„Das allgemeine Kennzeichen der dissoziativen oder Konversionsstörungen besteht in teilweisem oder völligem Verlust der normalen Integration der Erinnerung an die Vergangenheit, des Identitätsbewusstseins, der Wahrnehmung unmittelbarer Empfindungen sowie der Kontrolle von Körperbewegungen. (...) Die Symptomeverkörpern häufig das Konzept der betroffenen Person, wie sich eine körperliche Krankheit manifestieren müsste. Körperliche Untersuchung und Befragungen geben keinen Hinweis auf eine bekannte somatische oder neurologische Krankheit. Zusätzlich ist der Funktionsverlust offensichtlich Ausdruck emotionaler Konflikte oder Bedürfnisse ..." 
Die ICD 10 ordnet dabei unterschiedliche Phänomene zu:

- F44.o dissoziative Amnesie

- F44.1 dissoziative Fugue

- F44.2 dissoziativer Stupor

- F44.3 Trance- und Besessenheitsstörung

- F44.4-F44.7 dissoziative Störungen der Bewegung und der Sinnesempfindung

- F44.4 dissoziative Bewegungsstörung

- F44.5 dissoziative Krampfanfälle

- F44.6 dissoziative Sensibilitäts- und Empfindlichkeitsstörungen

- F44.7 dissoziative Störungen (Konversionsstörungen), gemischt

- F44.8 sonstige dissoziative Störungen (Konversionsstörungen)

- F44.80 Ganser Syndrom

- F44.81 multiple Persönlichkeitsstörung

- F44.82 vorübergehende dissoziative Störung

- F44.88 sonstige nicht näher bezeichnete dissoziative Störungen (Konversionsstörungen)

- F44.9 nicht näher bezeichnete dissoziative Störungen (Konversionsstörung)

Forschung der letzten Zeit in Bezug auf dissoziative Störungen zielte in der Mehrheit der publizierten Studien auf kognitive Störungen bzw. Symptome im Bereich von Wahrnehmung, Mnestik und Bewusstsein, wie beispielsweise Störungen von Orientierung und Kurzzeitgedächtnis, Depersonalisation und Derealisation ab. In der Diagnostik wurden zu diesem Schwerpunkt Fragebögen wie die Dissociative Experiences Scale (Wiener 1992) sowie spezifische Experteninterviews wie der Dissociative Disorders Interview Schedule (Yargic et al. 1998) entwickelt.

In diesem Modell gehen viele Autoren von einer engen kausalen Beziehung zwischen Belastungen - insbesondere längerer Exposition gegenüber sexueller oder sexualisierter Gewalt in Kindheit oder Erwachsenenalter - und dissoziativen Phänomenen aus (Avdibegovic u. Sinanovic 2006; Bailey et al. 2007; Berger et al. 1994), die in diesem Zusammenhang oft mit z.B. Essstörungen (Berger et al. 1994) auftreten, allerdings ohne dass alle Betroffenen an einer dissoziativen Störung leiden. Epidemiologisch orientiere Publikationen zeigen dabei, dass dissoziative Phänomene auch in der Allgemeinbevölkerung relativ häufig beobachtet werden (Aderibigbe et al. 2001; Elmore 2000) und daher wahrscheinlich nicht allein posttraumatisch oder im Rahmen normativer Psychopathologie erklärt werden können. Viele Autoren fanden außerdem hohe Unterschiede in den Prävalenzen bei unterschiedlichen ethnischen Cruppen, die als Hinweis auf kulturelle Faktoren interpretiert werden können.

Trance als weiteres Beispiel aus dieser Cruppe ist in der transkulturellen Psychiatrie im Prinzip ein Sammelbegriff für ein breites Spektrum unterschiedlicher Veränderungen des Wachbewusstseins. Bereits die (ursprünglich aus Österreich stammende) Ethnologin Erika Bourguignon demonstrierte in einer 
bis heute richtungweisenden Analyse vorhandener Daten (Bourguignon 1973; siehe auch Jones u. Wessely 2004), dass Trancezustände in den meisten Kulturen vorkommen und in der Regel nicht als pathologisch zu sehen sind, sondern wichtige Funktionen erfüllen können. Im Sinne der transkulturellen Psychiatrie (und in Übereinstimmung mit der ICD 10) sind dabei Trancen (oder im weiteren Sinne veränderte Wachbewusstseinszustände [VWBs]) nur dann als pathologisch und potenziell behandlungsbedürftig zu sehen, wenn sie in der entsprechenden Kultur als krankhaft interpretiert würden. Dies soll allerdings eine sorgfältige Differenzialdiagnostik im Zweifelsfall nicht ausschließen. Als Beispiele sind Besessenheitstrancen zu sehen, in denen die Erfahrung - wie beispielsweise in Umbanda - als erwünscht gesehen und ,eingeladen" wird, dabei jedoch meist vorgegebenen Regeln folgt und durch kulturgesteuerte Lernprozesse sowie Helfer in der Umgebung unterstützt wird. Erst wenn diese zu einer erheblichen Beeinträchtigung führen und nicht mehr kulturell vorgegebenen Rahmenbedingungen folgen, wird ein Experte - allerdings aufgrund der metaphysischen bzw. religiösen Interpretation eher ein Heiler - in der jeweiligen Tradition (Bourguignon 1973; Goodman et al. 1982) beigezogen. Die Entwicklung neurophysiologischer und bildgebender Verfahren ermöglichte in den letzten Jahren wichtige Beiträge zum verbesserten Verständnis biologischer Prozesse im Rahmen von veränderten Wachbewusstseinszuständen (VWZs) (Avner 2006) und legt eher ein Spektrum möglicher VWZs als ein einheitliches neurophysiologisches Muster nahe.

Trance wird in vielen Kulturen dabei durch Experten eingesetzt (im tungusisch-altaischen Raum beispielsweise durch Schamanen, die ein jahrelanges Training durchlaufen), um Heilungsprozesse beispielsweise durch die Rückholung einer „verlorenen Seele“ zu ermöglichen (Neill 1993). Hierbei werden in selteneren Fällen auch wachbewusstseinsverändernde Pflanzen eingesetzt. Meist erfolgt die Induktion aber durch andere Techniken (Goodman 1988). Kulturabhängig sind diesen Spezialisten dabei verschiedene Formen der Trance vorbehalten. In anderen Kulturen oder religiösen Cruppierungen, wie beispielsweise den Pfingstlerbewegungen, ist die Trance als Kommunikation mit Gott oder den Göttern Teil allgemein geteilter, religiöser Erfahrung. Wird Trance, wie etwa bei Formen der Besessenheitstrance, in denen „Teufel“ oder „Djinns“ unerwünscht vom Betroffenen Besitz ergreifen, als Bedrohung erlebt, liegt ein - im Sinne auch der WHO Definition (F 44.3) - pathologischer Zustand vor und Experten wie Priester werden beigezogen, um den unerwünschten Gast zu vertreiben. Eine Fallstudie aus unserer Kultur und Gegenwart ist dabei der Fall „Anneliese Michel“ (Goodman 1981), bei dem ein junges Mädchen während eines Exorzismus verstarb.

Idioms of Distress im Sinne des DSM-5 können als mögliches Modell angewandt werden, ohne Trancephänomene generell erklären zu können. Eine Gleichsetzung mit psychopathologischen Phänomenen erscheint aber ebenfalls durch das Vorliegen eines solchen „Idioms“ nicht gerechtfertigt, da oft kein Behandlungsauftrag oder Wunsch z.B. an medizinische Versorgung, 
sondern eher ein soziales Interaktionsphänomen vorliegt, dass oft mit Zuwendung im sozialen Netzwerk beantwortet und „gelöst“ wird.

Eine genaue Analyse der Bedeutung und Rahmenbedingungen von Trancephänomenen im kulturellen Gesamtkontext ist hier ebenfalls einer vereinfachten und aus dem Kontext herausgenommenen Einschätzung vorzuziehen.

\subsection{Behandlung und Behandlungsbedarf}

Unter Berücksichtigung aller bisherigen Modelle erscheint es sinnvoll, davon auszugehen, dass dissoziative Phänomene ein Spektrum von Phänomenen beschreiben, das von normalen Alltagsphänomenen zu Symptomen schwerwiegender und für den Betroffenen belastenden Erkrankungen unterschiedlicher Ätiologie führt, die nicht sicher durch ein einheitliches Modell integriert werden können. Am einen Ende stehen dabei gängige Alltagserfahrungen wie das Tagträumen in langweiligen Alltagssituationen, aus denen man sich rückorientieren muss. Am anderen Ende stehen indes tiefergehende und unter Umständen schwer kontrollierbare Formen, wie zum Beispiel die im öffentlichen Diskurs beliebte, in der Praxis aber eher seltenere, dissoziative Identitätsstörung (Multiple Persönlichkeitsentwicklung) (International Society for the Study of Trauma and Dissociation 2011; Abrams 1983) als Folge des Überlebens in einer traumatischen Familienumgebung oder die bereits angesprochenen, pathologischen Formen der Besessenheitstrance.

Dissoziative Reaktionen können im Kontext von Belastungen auch als ursprünglich adaptiv gesehen werden, da sie als „dritte Option“ in einer Bedrohungssituation, in der dem Betroffenen z.B. bei anhaltendem Missbrauch weder Kampf noch Flucht möglich ist, die physisch oder psychisch zu belastende bewusste Erfahrung aus dem bewussten Erleben oder Erleiden abspaltet. Sie sind daher in vielen Situationen als „normale“ Reaktion auf ungewöhnliche Situationen und Belastungen zu sehen (Wenzel 2007). Erst wenn sie nach Abklingen der Gefahrensituation auch durch harmlose Alltagssituationen "getriggert“ werden oder Teil einer komplexeren, „, neurotischen“ Entwicklung werden und erheblich mit diesen interferieren, z.B. ein Lernen in der Schule unmöglich machen, werden sie zu einem Problem das Behandlung erfordert. Sie sind in diesem Zusammenhang auch als Zeichen einer möglichen Belastungssituation oder Traumatisierung ernst zu nehmen.

Es überrascht daher nicht, dass dissoziative Phänomene bzw. Symptome weiter als mögliche oder „assoziierte“ Symptome im Rahmen anderer psychischer Reaktionen und Erkrankungen, besonders bei der posttraumatischen Belastungsstörung (PTBS, ICD 1o F 43.1) oder wie bereits beschrieben bei sexueller Gewalt, im Kontext mit Borderlinestörungen (Chu u. Dill 1991) oder Essstörungen (Berger et al. 1994) auftreten. Dissoziative Reaktionen bei besonderen Belastungserlebnissen, die im Rahmen einer akuten Belastungsreaktion oder 
als eigenständige Phänomene auftreten, werden von einigen Autoren als Zeichen nicht erfolgreichen Copings und als Prädiktor einer späteren PTBS gesehen (Bryant 2003).

Obwohl eine Psychotherapie als wichtigster Aspekt einer Behandlung zu sehen ist, ist die Einbindung in einen erweiterten interdisziplinären und methodenintegrativen Ansatz nach sorgfältiger Differenzialdiagnostik besonders bei schweren Belastungen als Auslöser der Symptomatik dringend erforderlich (Spitzer et al. 1996). Das kulturabhängige Ausdrucksverhalten unter Belastung („Idiom of Distress“) und der kulturelle Rahmen sollten dabei besonders berücksichtigt werden. Aus dem kulturellen Kontext angebotene Heilungsmodelle sind in diesem Zusammenhang als Möglichkeit der transkulturellen Praxis zu erheben und falls sinnvoll und möglich einzubinden, sofern ein sinnvoller Behandlungsauftrag abgeleitet werden kann.

\subsection{Forensische Relevanz}

Die bisherige Übersicht hat deutlich gemacht, dass die derzeit (noch) aktuelle ICD Klassifikation in Bezug auf diagnostische Kategorien in diesem Punkt mit Vorsicht zu sehen ist und sehr unterschiedliche Phänomene vor dem Hintergrund biologischer, erfahrungsabhängig-individueller und kultureller Faktoren vorliegen können. Eine sorgfältige Analyse dieser Faktoren im Einzelfall ist daher vereinfachenden Generalisierungen auf der Basis der unklaren Begriffsverwendungen oder der Beschränkung auf spezifische Fragebogeninstrumente vorzuziehen (Brand et al. 2006; Sparr u. Atkinson 1986). Die Einbeziehung des DSM kann zumindest im Bereich transkultureller Fragestellungen wahrscheinlich zu einer deutlichen Verbesserung führen. Fragestellungen können dabei sowohl Täter wie Opfer (Werner u. Werner 2008) betreffen. In Bezug auf Fragestellungen bei Opfern kann das Istanbul Protokoll der Vereinten Nationen als Beispiel für eine differenzierte Richtlinie zur forensischen Begutachtung angeführt werden (United Nations 2001; Perera u. Verghese 2011).

- Einschränkung von Bewusstsein, Auffassung, Mnestik, Urteilsfähigkeit sowie zeitlicher und örtlicher Orientierung

- Einschränkung motorischer Funktionen im Rahmen eines funktionellen Geschehens

- Abhängigkeit dieser Beeinträchtigung von situativen Faktoren

- als Hinweis auf (über längere Zeit anhaltende) Erfahrung sozialer Gewalt, besonders sexueller Natur

- als kulturspezifisches Phänomen in unterschiedlichem Kontext, beispielsweise bei traumatisierten Flüchtlingen in Asylverfahren

- Beeinträchtigung und Störung von Wohlbefinden und spezifischen oder Alltagsaktivitäten 


\subsection{Differenzialdiagnose}

Auf Differenzialdiagnostik und Behandlung kann im Rahmen dieses Artikels nur kurz eingegangen werden. Auch bei hirnorganischen und zahlreichen neurologischen Krankheitsbildern, beispielsweise bei hirnlokalen Psychosyndromen, Schädelhirntrauma (Broomhall et al. 2009) und Epilepsien (Alper et al. 1997), oder bei Substanzgebrauch können sowohl dissoziative Symptome als auch diesen ähnliche Symptome auftreten, sodass eine sorgfältige differenzialdiagnostische Abklärung auch bei scheinbar eindeutig psychologischen Auslösern dringend indiziert ist.

\section{Zusammenfassung}

Dissoziative Phänomene sind Teil alltäglicher psychologischer Mechanismen. Dissoziative Störungen entstehen meist in Belastungssituationen und sind in ihrer Ausprägung oft kulturabhängig zu verstehen. Eine Differenzialdiagnostik zur Abgrenzung von anderen, z.B. hirnorganischen Auslösern oder Symptomen, ist allerdings wesentlich.

Dieser Beitrag basiert auf einem Übersichtsartikel der Autoren in Spectrum Psychiatrie (Zhu et al. 2011). Mit freundlicher Genehmigung der MedMedia Verlag und Mediaservice GmbH.

\section{Literatur}

Abrams S (1983) The multiple personality: a legal defense. Am I Clin Hypn 25(4), 225-31

Aderibigbe YA, Bloch RM, Walker WR (2001) Prevalence of depersonalization and derealization experiences in a rural population. Soc Psychiatry Psychiatr Epidemiol 36(2), 63-9

Alper K, Devinsky 0, Perrine K, Luciano D, Vazquez B, Pacia S. Rhee E (1997) Dissociation in epilepsy and conversion nonepileptic seizures. Epilepsia 38(9), 991-7

Avdibegovic E, Sinanovic 0 (2006) Consequences of domestic violence on women's mental health in Bosnia and Herzegovina. Croat Med I 47(5), 730-41

Avner IR (2006) Altered states of consciousness. Pediatr Rev 27(9), 331-8

Bailey HN, Moran G, Pederson DR (2007) Childhood maltreatment, complex trauma symptoms, and unresolved attachment in an at-risk sample of adolescent mothers. Attach Hum Dev 9(2), 139-61

Berger D, Saito S, Ono Y, Tezuka I, Shirahase J, Kuboki T, Suematsu H (1994) Dissociation and child abuse histories in an eating disorder cohort in Japan. Acta Psychiatr Scand 90(4), 274-80

Bourguignon E (1973) Religion, altered states of consciousness, and social change. Ohio State University Press Columbus

Brand BL, McNary SW, Loewenstein RJ, Kolos AC, Barr SR (2006) Assessment of genuine and simulated dissociative identity disorder on the structured interview of reported symptoms. I Trauma Dissociation 7(1), 63-85

Broomhall LG, Clark CR, McFarlane AC, O'Donnell M, Bryant R, Creamer M, Silove D (2009) Early stage assessment and course of acute stress disorder after mild traumatic brain injury. I Nerv Ment Dis 197(3), 178-81

Bryant RA (2003) Acute stress reactions: can biological responses predict posttraumatic stress disorder? CNS Spectr 8(9), 668-74

Chu JA, Dill DL (1991) Dissociation, borderline personality disorder, and childhood trauma. Am I Psychiatry 148(6), $812-3$ 
Degkwitz R (1975) World Health Organization. Deutsche Gesellschaft für Psychiatrie und Nervenheilkunde. Diagnosenschlüssel und Glossar psychiatrischer Krankheiten: deutsche Ausgabe der internationalen Klassifikation der WHO, ICD (International classification of diseases). 4., korregierte und erweiterte Auflage. Springer-Verlag Berlin New York

Elmore IL (2000) Dissociative Spectrum Disorders in the Primary Care Setting. Prim Care Companion I Clin Psychiatry 2(2), 37-41

Goodman FD (1981) The exorcism of Anneliese Michel. 1. Auflage. Doubleday Garden City N.Y.

Goodman FD, Henney JH, Pressel E (1982) Trance, healing, and hallucination: three field studies in religious experience. R.E. Krieger Pub. Co. Huntington N.Y.

Goodman FD (1988) Ecstasy, ritual and alternate reality: religion in a pluralistic world. Indiana University Press Bloomington

Hare E (1991) The history of ,nervous disorders' from 1600 to 1840, and a comparison with modern views. $\mathrm{Br}$ ) Psychiatry 159; 37-45

International Society for the Study of Trauma and Dissociation (2011) Guidelines for treating dissociative identity disorder in adults, third revision: summary version. I Trauma Dissociation 12(2), 188-212

Isaac M, Chand PK (2006) Dissociative and conversion disorders: defining boundaries. Curr Opin Psychiatry 19(1), 61-6

Jones E, Wessely S (2004) Hearts, guts and minds: somatisation in the military from 1900. I Psychosom Res 56(4), 425-9

Mace CI (1992) Hysterical conversion. A history. Br I Psychiatry 161, 369-77

Neill JR (1993) How psychiatric symptoms varied in World War I and II. Mil Med 158(3), 149-51

Perera C, Verghese A (2011) Implementation of Istanbul Protocol for effective documentation of torture review of Sri Lankan perspectives. I Forensic Leg Med 18(1), 1-5

Scull AT (2009) Hysteria: the biography. Oxford University Press Oxford New York

Sparr LF, Atkinson RM (1986) Posttraumatic stress disorder as an insanity defense: medicolegal quicksand. Am I Psychiatry 143(5), 608-13

Spitzer C, Freyberger HJ, Kessler C (1996) Hysteria, dissociation and conversion. A review of concepts, classification and diagnostic instruments. Psychiatr Prax 23(2), 63-8

United Nations (2001) Office of the High Commissioner for Human Rights. Istanbul Protocol: manual on the effective investigation and documentation of torture and other cruel, inhuman or degrading treatment or punishment. United Nations New York

van Duijl M, Nijenhuis E, Komproe IH, Gernaat HB, de Jong IT (2010) Dissociative symptoms and reported trauma among patients with spirit possession and matched healthy controls in Uganda. Cult Med Psychiatry 34(2), $380-400$

Veith I (1965) Hysteria: the history of a disease. University of Chicago Press Chicago

Wenzel T (2007) Torture. Curr Opin Psychiatry 20(5), 491-6

Werner J, Werner MC (2008) Child sexual abuse in clinical and forensic psychiatry: a review of recent literature. Curr Opin Psychiatry 21(5), 499-504

Wiener A (1992) The Dissociative Experiences Scale. Am J Psychiatry 149(1), 143-4

Yargic LI, Sar V, Tutkun H, Alyanak B (1998) Comparison of dissociative identity disorder with other diagnostic groups using a structured interview in Turkey. Compr Psychiatry 39(6), 345-51

Zhu LI, Fabian F, Wenzel T (2011) Dissoziation und dissoziative Erkrankungen im Kontext der transkulturellen Psychatrie. Spectrum Psychiatrie 1, 36-38 